\title{
MRI evaluation of tissue iron burden in patients with $\beta$-thalassaemia major
}

\author{
Maria I. Argyropoulou • Loukas Astrakas
}

Received: 13 February 2007 /Revised: 7 May 2007 / Accepted: 31 May 2007 / Published online: 21 August 2007

(C) Springer-Verlag 2007

\begin{abstract}
Thalassaemia major is a hereditary haemolytic anaemia that is treated with multiple blood transfusions. A major complication of this treatment is iron overload, which leads to cell death and organ dysfunction. Chelation therapy, used for iron elimination, requires effective monitoring of the body burden of iron, for which serum ferritin levels and liver iron content measured in liver biopsies are used as markers, but are not reliable. MRI based on iron-induced $\mathrm{T} 2$ relaxation enhancement can be used for the evaluation of tissue siderosis. Various MR protocols using signal intensity ratio and mainstream relaxometry methods have been used, sometimes with discrepant results. Relaxometry methods using multiple echoes achieve better sampling of the time domain in which relaxation mechanisms take place and lead to more precise results. In several studies the MRI parameters of liver siderosis have failed to correlate with those of other affected organs, underlining the necessity for MRI iron evaluation in individual organs. Most studies have included children in the evaluated population, but MRI data on very young children are lacking. Wider application of relaxometry methods is indicated, with the establishment of universally accepted MRI protocols, and further studies, including young children, are needed.
\end{abstract}

Keywords $\beta$-Thalassaemia $\cdot$ MRI $\cdot$ Iron $\cdot$ Relaxometry

M. I. Argyropoulou $(\bowtie) \cdot$ L. Astrakas

Radiology Department, Medical School, University of Ioannina, Ioannina GR-45110, Greece

e-mail: margyrop@cc.uoi.gr

\section{Introduction}

$\beta$-Thalassaemia major is a hereditary anaemia characterized by ineffective erythropoiesis and haemolysis [1]. The underlying mechanism is defective production of haemoglobin $\beta$ chains, resulting in excess of $\alpha$-chains, which are unstable and precipitate to form intracellular inclusion bodies [2, 3]. This excessive intracellular deposition of $\alpha$-chain material is responsible for accelerated apoptosis of the erythroid precursors and for peripheral haemolysis of the erythrocytes [3]. By the age of 3 months, severe anaemia develops leading to increased intestinal iron absorption. To maintain haemoglobin at a level of 10-12 g/dl, patients suffering from $\beta$-thalassaemia major need to be given repeated blood transfusions [1]. A major drawback of this treatment is transfusion siderosis, which, in association with the increased intestinal iron absorption, apoptosis of the erythroid precursors and peripheral haemolysis, leads to iron overload [1].

Iron is ubiquitous in eukaryotic organisms. It is essential for cellular survival and proliferation and for haemoglobin synthesis [4, 5]. Human demands for iron are covered partly by intestinal absorption, but mainly from the recycling of iron from old or abnormal erythrocytes phagocytized by macrophages [6]. Iron released from macrophages binds mainly to transferrin, but also to citrate and albumin to form non-transferrin-bound iron (NTBI) which is a toxic form [7-9]. Extracellular iron bound to transferrin enters the cell via transferrin receptors by a process of endocytosis. Once iron is released into the cytoplasm, it enters a poorly defined cellular compartment termed the labile iron pool (LIP). Iron in this compartment is loosely bound and therefore is highly toxic [10]. Intracellular iron that is not needed for immediate use is stored in the form of ferritin which consists of an 
apoprotein storing up to 4,500 atoms of iron (loading factor $4,500)[5,11]$, and is not cytotoxic. When ferritin storage capacity is exceeded the LIP increases and haemosiderin is generated from ferritin denaturation [10]. Iron in the form of haemosiderin is thought to be more cytotoxic [10]. The iron in the LIP is in both the ferrous $\left(\mathrm{Fe}^{2+}\right)$ and ferric $\left(\mathrm{Fe}^{3+}\right)$ forms [12]. $\mathrm{Fe}^{2+}$ reacts with hydrogen and lipid peroxides and generates highly toxic hydroxyl and lipid radicals (Fenton reaction) that damage cellular membranes, proteins and nucleic acids [4]. Iron overload, just as lack of cellular iron, may lead to cell death and organ dysfunction.

Iron overload is a major cause of morbidity and mortality in $\beta$-thalassaemia major [1]. Iron accumulates initially in the reticuloendothelial system (bone marrow, spleen, liver) and then in the hepatocytes, the heart (myocytes) and the endocrine glands $[1,9,13]$. In contrast to the reticuloendothelial cells, the turnover of iron in the hepatocytes, myocytes and endocrine glands is very low [9]. Chelation therapy has been used to eliminate excess iron $[1,7]$. Chelatable iron is derived from the catabolism of haemoglobin in macrophages [14]. Chelators remove NTBI from the plasma, but they do not interact with ferritin and haemosiderin at clinically relevant rates [10, 15]. Desferrioxamine (DFO), which is the chelating agent most widely used over the last 30 years, requires parenteral administration. It removes mainly extracellular iron and only a fraction of the intracellular LIP iron [14, 15]. Deferiprone is an oral iron chelator that penetrates the cellular membrane and chelates intracellular species of toxic iron [16]. Cellular uptake of chelators takes place at different rates in different cells [15]. DFO is of low toxicity, mainly affecting the optic, auditory and skeletal systems [7]. Skeletal changes develop mainly due to the toxic effects of DFO on the growth cartilage and are manifested as disproportionate truncal shortening with loss of seated height [7]. Adverse effects of deferiprone are arthralgia, gastrointestinal symptoms, elevated liver enzymes and rarely agranulocytosis $[7,16]$.

The effective management of patients, and especially of children, with thalassaemia requires optimal monitoring of the toxic effects of both iron overload and excessive chelation therapy. Serum ferritin has been widely used as a surrogate marker and a target ferritin level of $1,000 \mu \mathrm{g} / \mathrm{l}$ is generally recommended [7, 17]. However, serum ferritin represents only $1 \%$ of the total iron pool, and as an acutephase protein it is not specific because the levels can be raised in inflammation (e.g. hepatitis) and liver damage [7, 18, 19]. Chronic liver inflammation is not rare in patients with thalassaemia, since over $40 \%$ of them have positive anti-hepatitis $\mathrm{C}$ virus (HCV) antibodies and more than $50 \%$ have chronic (persistent or active) hepatitis [20, 21]. Liver iron concentration (LIC) measured on needle biopsy is currently considered the gold standard for the evaluation of siderosis $[7,18,19]$. However, needle biopsy is an invasive technique, it is not easily repeatable and the accuracy of the resulting LIC measurement is greatly affected by hepatic inflammation-fibrosis and uneven iron distribution [22]. Furthermore, it appears that cardiac iron overload, which is the leading cause of death in thalassaemia, cannot be predicted from the degree of liver siderosis, probably because of differences in iron kinetics between liver and heart cells [9]. The use of non-invasive techniques for monitoring iron overload in each of the affected organs would be preferable, and to this end MRI has been used increasingly over the last two decades [19, 22-44]. Other non-invasive methodologies are too complex, too costly and not readily available (i.e. magnetic susceptometry), or lack imaging capability (i.e. magnetic resonance spectroscopy) $[45,46]$.

\section{MRI assessment of tissue iron}

\section{Relaxation theories}

MRI evaluation of tissue iron overload is based on T2 relaxation enhancement induced from the interactions between high-molecular-weight iron complexes such as ferritin, haemosiderin and ferrioxamine with water molecules $[5,47,48]$. Ferrioxamine is produced after cellular uptake of DFO, and in extrahepatic tissues, where there is no active excretion and it may remain for days [7]. Ferrioxamine-based agents have been used in the past as contrast media because of their T2* effect [49]. "Inner sphere" and "outer sphere" relaxation theories have been proposed to explain the $\mathrm{T} 2$ relaxation enhancement induced in siderotic tissues [50-55]. According to the inner sphere theory, iron electron spins enhance the relaxation of protons of water molecules buried in iron-containing proteins. Enhanced relaxation of the bound water protons is then transferred to the free water protons by water or proton exchange [48, 56, 57]. According to the outer sphere theory, magnetic field gradients induced at the periphery of iron-containing proteins create loss of phase and relaxation enhancement of free water protons diffusing in the neighbourhood [53, 54].

\section{MR methodologies}

MR methods for assessing tissue iron can be separated into two groups: signal intensity ratio (SIR) methods and relaxometry methods. Various techniques have been described, including: (a) methods measuring SIR based on T2-weighted (spin-echo) or T2*-weighted (gradient-echo) sequences [19, 23-26, 30, 58-62], (b) relaxometry methods measuring absolute $\mathrm{T} 2$, (c) relaxometry methods measuring 
absolute $\mathrm{T} 2 *$, and (d) hybrid relaxometry methods [30-32, 34-37, 39-43, 58, 63].

\section{SIR methods}

These have been used for the study not only of the liver but also of other organs such as the spleen, pancreas, pituitary gland, bone marrow and abdominal lymph nodes [19, 23, 25, 27, 30, 58-62]. For SIR assessment the signal intensity of the target organ is divided by the signal intensity of a reference tissue (e.g. fat, muscle) or noise. Signal intensity measurements are performed in the same slice by using the region-of-interest (ROI) method. For large organs such the liver, spleen and pancreas more than one ROI is used, positioned in areas lacking vascular structures and movement artefacts $[19,23,25,27,30,58,61,62]$. The mean signal intensity from the different ROIs is then divided by the signal intensity of the reference tissue. One ROI is used for signal intensity measurement of the pituitary gland, bone marrow, abdominal lymph nodes and the reference tissue [58-60]. For SIR evaluation of the liver various reference tissues have been used, of which paraspinal muscle appears to be the best choice because it combines good sensitivity with minimum intersite variability [19, 64]. A disadvantage of the SIR methods is that in most cases they use only one echo time (TE) and thus lose their detection sensitivity in tissues with heavy siderosis, where transverse relaxation is much faster than the TE. This occurs particularly in the liver at the upper range of LIC values where signal intensities are widely dispersed [39]. Gandon et al. [19], by using an algorithm that combined signal intensity ratios from multiple sequences with different TEs, achieved extension of the detection range up to about $21 \mathrm{mg}$ Fe per gram dry liver tissue, with sensitivity and specificity similar to those of biochemical analysis.

\section{$T 2$ relaxometry methods}

These assess T2 relaxation time or R2 (1/T2) by using the Carr-Purcell-Meiboom-Gill (CPMG) spin-echo sequence, which employs multiple (2-32) equidistant refocusing $180^{\circ}$ pulses, each followed by an echo $[65,66]$. Most scanners, by using a pixel-by-pixel, log-linear fitting model, automatically derive the corresponding T2 maps. Signal intensity measurements in the T2 maps correspond to the mean T2 relaxation time of the included voxels [67]. To avoid motion artefacts from respiration, studies of abdominal organs should be performed with respiratory triggering. To this end a pressure-sensitive pad is attached to the abdomen and depending on the patient's respiratory cycle a delay time is chosen. Data acquisition takes place at the end-expiration phase $[39,40]$. During cardiac MRI, in addition to respiratory triggering, cardiac triggering is necessary. To obtain a minimum repetition time (TR) of 2,000 $\mathrm{ms}$ two to three $\mathrm{R}-\mathrm{R}$ intervals should be allowed between successive excitations [39, 40]. The mid-systole phase and a cardiac trigger delay of around 250-300 ms are usually chosen to image the myocardium with sufficient thickness for the best signal intensity measurements [39, 40]. A pseudo four-chamber or short-axis view of the heart is generally used. A T2 relaxometry method that received FDA approval for clinical liver iron estimation has recently been developed by St. Pierre et al. [44]. This method uses multiple T2-weighted single spin-echo sequences with different TEs acquired in half-Fourier mode to reduce acquisition time. The calculated mean R2 values combined with LIC values, obtained from liver biopsies, are used to create calibration curves.

\section{T2* relaxometry methods}

These evaluate $\mathrm{T} 2 *$ or $\mathrm{R} 2 *(1 / \mathrm{T} 2 *)$ by using multiple gradient-echo sequences with different TEs. These methods have been developed to further accelerate acquisition, in order to increase sensitivity and eliminate artefacts related to respiration or cardiac motion. To obtain $\mathrm{R} 2 *(1 / \mathrm{T} 2 *)$ values, the signal decay curve is usually fitted with an exponential model: $\mathrm{S}=\mathrm{S}_{0} \mathrm{e}^{-\mathrm{TE} / \mathrm{T} 2^{*}}$, where $\mathrm{S}$ is the net image signal intensity, $\mathrm{TE}$ is the echo time and $\mathrm{S}_{0}$ is a constant [68]. T2* relaxometry methods have been used mainly for myocardial iron assessment and cardiac gating is always applied [35, 42, 68-71]. Breath-hold sequences have greatly eliminated motion artefacts $[35,42,68]$.

\section{Hybrid relaxometry methods}

Hybrid approaches have been applied in high fields and measure both R2 and R2* to calculate the inhomogeneity factor $\mathrm{R}^{\prime}=\mathrm{R} 2 *-\mathrm{R} 2[34,72,73]$. These approaches assume that R2' $^{\prime}$ is more specific to mechanisms of relaxation related to iron than $\mathrm{R} 2[34,73]$.

\section{Comparison of the MRI methodologies}

\section{SIR versus relaxometry}

SIR methods require shorter acquisition times but lack a wide range of iron assessment [63]. Relaxometry methods, mainly the $\mathrm{T} 2 *$ method, by using multiple echoes create in- and out-of-phase effects between water and fat transverse magnetization (Fig. 1) [35]. Relaxometry methods, although taking longer, are preferable because they achieve a better sampling of the time domain in which relaxation mechanisms take place and lead to more precise results [63]. 


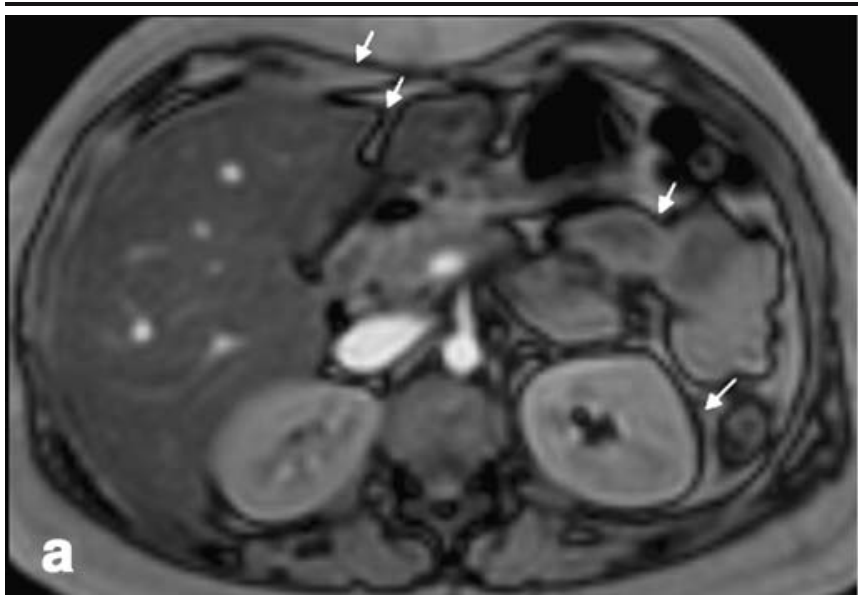

Fig. 1 A 15-year-old male with $\beta$-thalassaemia major. Axial scan with a multiecho gradient-echo sequence (TR/TE 150/1.07, 2.14, 3.21, $4.28,5.35,6.42,7.49,8.56,9.63,10.7,11.77,12.84,13.91,14.98$, $16.05,17.12,18.19,19.26,20.33,21.4 \mathrm{~ms}$; flip angle $35^{\circ}$ ). a Second

\section{T2, T2* and hybrid methods}

There is no general consensus on which relaxometry method (spin-echo or gradient-echo) or index (R2, R2*, $\mathrm{R} 2$ ') is best for tissue iron quantification. Theoretically, $\mathrm{R} 2 *$ and R2' are more sensitive than R2 to iron-induced field inhomogeneities. Gradient-echo sequences evaluating R2* are preferred for cardiac relaxometry, because of their short acquisition time, but gradient-echo sequences are more prone to artefacts and $\mathrm{R} 2 *$ is more dependent on factors unrelated to iron, such as susceptibility artefacts from the lungs and the blood oxygenation level-dependent (BOLD) effect $[35,74]$. According to the BOLD effect, the T2* decreases as the concentration of intracapillary deoxyhaemoglobin increases [74]. A pronounced BOLD effect may be observed in early diastole and results in signal loss $[75,76]$. Data collection during mid-systole helps to overcome BOLD effect interference with accurate $\mathrm{T} 2 *$ measurements of the myocardium. In liver and pituitary studies both $\mathrm{T} 2$ and $\mathrm{T} 2 *$ have been assessed. T2* is more sensitive to low iron content, but studies in the liver show that these methods suffer from inaccuracies at high iron concentrations [77]. Furthermore, in high fields susceptibility artefacts from the sphenoid sinus are more pronounced with gradient-echo sequences and may lead to inaccurate measurements of pituitary siderosis [43].

\section{MRI studies of individual iron overloaded organs}

The degree of siderosis, the crystalline structure of ferritin, the rate of iron elimination under chelation therapy and the degree of ferrioxamine formation are all organ-specific $[7,15,18$, 78]. All these parameters may be responsible for differences in the $\mathrm{T} 2$ relaxation enhancement induced in the various

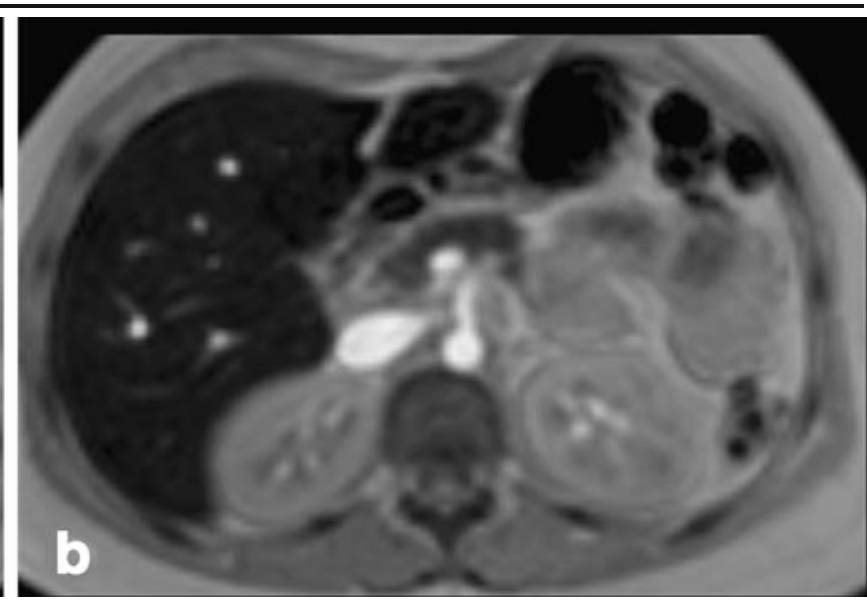

echo shows pixel annulation (arrows) due to out-of-phase phenomena, at the interfaces of abdominal organs and muscles with fat. b Fourth echo: no pixel annulation is observed because water and fat protons are in phase (courtesy of Dr. M. Douskou)

organs. Individual organs should be considered separately, and the effect of age on iron overload should be taken into account. Higher survival probabilities have been reported in patients with thalassaemia born in the last 30 years [79]. Patient compliance with treatment regimens and effective chelation therapy are thought to be the main factors associated with improved survival [79]. The combination of DFO, deferiprone and the new oral chelators is considered very promising, but will require effective monitoring by noninvasive methods [80]. An increasing number of studies have evaluated iron in the various affected organs by MRI [19, 22-44]. Many of these studies have included paediatric patients, but very few have investigated children younger than 10 years and none has included children younger than 5 years [17, 18, 28, 39, 43, 58, 60-62, 77, 81-84]. In addition, very few studies have evaluated the effect of age on individual organ siderosis and only one has evaluated and compared MRI data from younger and older patients with thalassaemia [28, 81, 82, 84]. Studies have tended to focus on specific organs.

Liver

For the MRI evaluation of liver siderosis both SIR and relaxometry techniques have been used (Fig. 2) [18, 23, 28, $32,44,68,85]$. R2 of the liver demonstrates a significant positive correlation with serum ferritin and LIC determined from liver biopsy material [18, 23, 32, 39, 40, 44, 68]. Comparative evaluation of hepatic R2 and R2* in ironoverloaded patients demonstrates that both parameters correlate closely with LIC [85]. The relationship of SIR with LIC and serum ferritin varies among studies [18, 30, 58]. In most studies R2 and SIR show a better correlation with LIC than with serum ferritin $[18,23,28,32,44,68$, 85]. This can be explained in part because the HCV- 


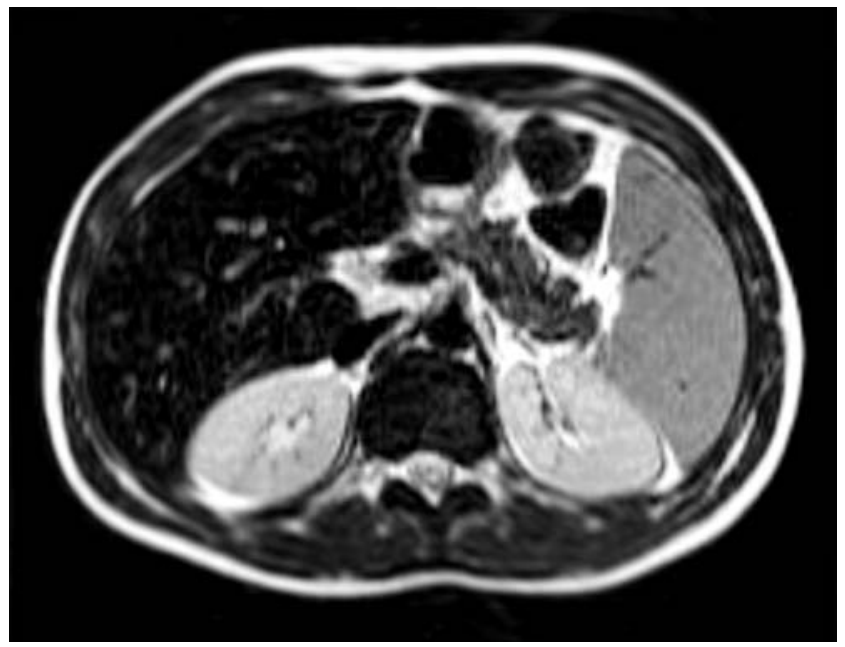

Fig. 2 A 17-year-old male with $\beta$-thalassaemia major. Axial scan, fourth echo of a multiecho spin-echo sequence (TR/TE: 2000/20, 40, $60,80,100,120,140,160 \mathrm{~ms}$ ), shows low-signal intensity in the liver, bone marrow of the spinal body and the pancreas suggesting iron overload

positive thalassaemia patients in the studies had higher serum ferritin levels than those who were HCV-negative $[77,86]$. Liver R2 shows no association with the hepatic inflammation histological activity index or the type of hepatitis (chronic persistent or chronic active), but is affected by hepatic fibrosis $[18,39,77]$.

In iron overload states, over $70 \%$ of body iron is found in the liver and LIC has been considered to be the best marker of total body iron burden $[7,18,19]$. Based on the good correlation between hepatic R2 or SIR and LIC a number of recent studies have tested the relationship between siderosis of the liver and other organs [28, 39, 40, 58-61, 87]. No correlation has been found between liver and pituitary siderosis $[28,58]$. With regard to the heart, a correlation with liver siderosis has been found only in cases of heavy myocardial iron deposition [39, 40]. This lack of correlation can probably be explained by differences in transferrin receptor concentration, iron kinetics, the crystalline structure of ferritin and the degree of organ inflammation or fibrosis [7, 78, 88-92]. Furthermore, under chelation therapy with
DFO intracellular paramagnetic ferrioxamine is formed, which exits slowly from cells unless there is an active excretion pathway as is present in the hepatocytes [88].

Young patients with thalassaemia studied longitudinally have shown absence of substantial improvement in the MR parameters of liver siderosis under different chelation therapy regimens [82]. This may be explained by the fact that liver siderosis progresses very fast in thalassaemia patients, and iron overload develops after only 2 years of transfusion therapy [93]. Therapy with the most widely used chelating agent is started at the age of about 3 years, and until growth is completed DFO should not exceed a dose of $40 \mathrm{mg} / \mathrm{kg}$ per day [7]. An early start to monitoring the progress of tissue iron deposition with MRI might be useful in deciding whether to begin chelation therapy at a younger age and when to introduce new chelating agents.

Heart

Cardiac failure is the leading cause of death from iron overload in patients with thalassaemia $[94,95]$. Thalassaemia patients on a regimen of regular blood transfusions who are not receiving chelation therapy, develop heart enlargement by the age of 10 years and heart failure by the age of 16 years [22]. Cardiomyopathy secondary to iron overload is potentially reversible with vigorous chelation therapy [7]. Myocardial iron has been evaluated by MRI using SIR and relaxometry techniques (Fig. 3) [22, 28, 39, 40, 68, 82, 9698]. In the prechelation era, post-mortem examination of patients with thalassaemia showed a close correlation between cardiac and hepatic iron concentrations. MRI studies have demonstrated discordant results regarding the relationship of myocardial iron with hepatic iron and serum ferritin [28, 39, 40, 68, 97]. Differences in iron kinetics and variations in chelation schemes may be responsible for the lack of correlation of the MRI-determined myocardial iron with that of the liver [88]. More active elimination of iron from the hepatocytes than from the myocytes may play a role in the absence of correlation in patients receiving chelation therapy $[28,39,68]$.
Fig. 3 A 16-year-old male with $\beta$-thalassaemia major. a T2 map of the short axis of the heart shows low values of the left ventricle (arrow) and the septum (arrowhead). b R2* (=1/T2*) map of the short axis of the heart shows high signal of the septum (asterisk). Note the reduction of motion artefacts and better delineation of the heart boundary in the R2* image (courtesy of Dr. M. Douskou)
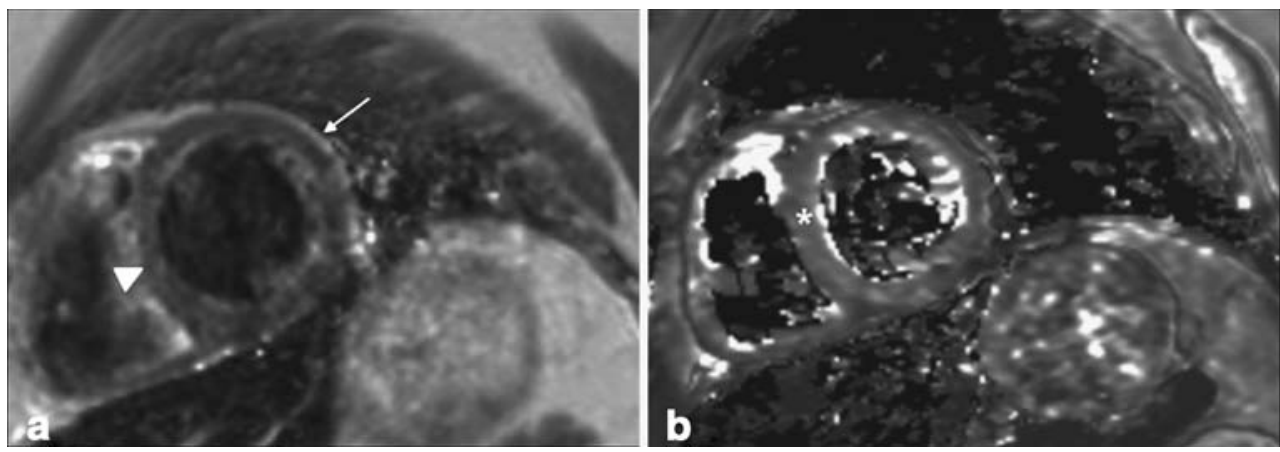


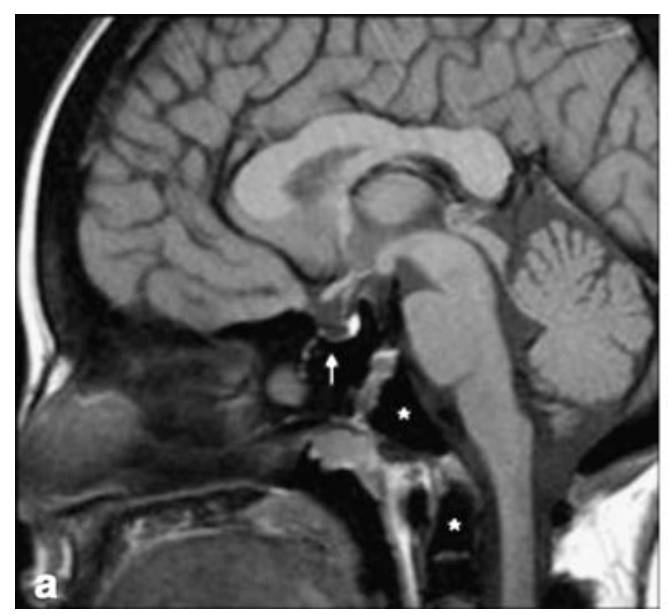

Fig. 4 The pituitary. a A 12-year-old male with $\beta$-thalassaemia major. Midsagittal T1-weighted (TR/TE 500/20 ms) scan shows low-signal intensity of the anterior pituitary lobe (arrow) and the bone marrow (asterisks) suggesting iron overload. The pituitary gland is small measuring $3.5 \mathrm{~mm}$ (normal for age $5.3 \pm 0.8 \mathrm{~mm}$ ) [112]. This patient

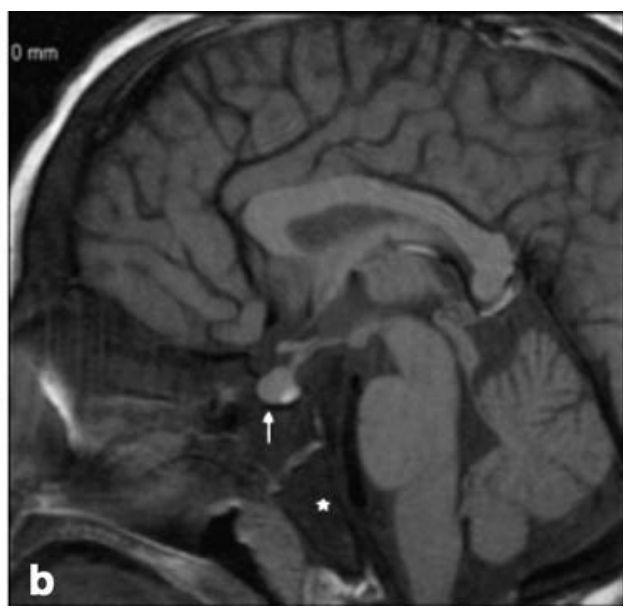

developed hypogonadotropic hypogonadism. b An 11-year-old male with $\beta$-thalassaemia major. Mid-sagittal T1-weighted (TR/TE 500/ $20 \mathrm{~ms}$ ) scan shows normal signal intensity of the anterior pituitary lobe (arrow) along with normal pituitary gland height $(6 \mathrm{~mm})$. The bone marrow (asterisk) shows low-signal intensity suggesting iron overload
Another important issue is whether myocardial iron evaluated by MRI could predict which patient will develop cardiac failure and arrhythmia. Thalassaemia patients with significantly reduced left ventricular ejection fraction (LVEF) have myocardial T2* values about $20 \mathrm{~ms}[22,37,38,68]$, although most patients with $\mathrm{T} 2 *$ values less than $20 \mathrm{~ms}$ have normal LVEF [68]. Furthermore, the relationship between myocardial R2 and LVEF is either non-existent or weak [22, 38-40]. Differences in cardiac functional indices among patients with similar MRI measurement of iron might also be explained by genetic factors that predispose to the more rapid development of cardiac failure when a critical level of myocardial siderosis is reached $[99,100]$.

Heterogeneous iron distribution in the myocardium has been demonstrated in histological studies, and this would suggest the necessity for global MRI estimation of myocardial iron [42, 101]. Pepe et al. [42], using T2* multiecho cardiac MRI, demonstrated that global T2* shows a close correlation with midseptal $\mathrm{T} 2 *$, suggesting that the latter may be used for a quick assessment of myocardial siderosis.

MR studies have shown that cardiac siderosis increases with age, but that siderosis progresses more slowly in the heart than in the liver $[28,82]$. Systematic MRI studies in children are necessary in order to evaluate the age at the start of cardiac iron overload.

\section{Pituitary gland and brain}

Increased iron deposition in the anterior pituitary gland is the cause of hypogonadotropic hypogonadism and growth hormone deficiency $[102,103]$. Iron accumulates in all five cell types of the adenohypophysis, but preferentially in the gonadotropin-secreting cells [102, 103]. Gonadotropin cell death due to iron toxicity is probably the cause of the decreased pituitary gland height observed in thalassaemia patients with hypogonadotropic hypogonadism (Fig. 4) [104]. MRI has been used to evaluate pituitary siderosis with SIR and relaxometry techniques [28, 43, 83, 105]. SIR and R2 findings show significant correlation with serum ferritin [83]. The very few studies evaluating simultaneously the pituitary and other solid organs have demonstrated no correlation between their MRI parameters of siderosis [28, 43, 81]. Pituitary siderosis appears to increase with age, but MR data are lacking for children under the age of 7 years $[28,81][84]$.

The single study assessing iron in the brain of thalassaemia patients by MRI [106] showed significantly higher R2 values in the cortex, the putamen and the caudate nucleus in patients than in controls. A lack of correlation between R2 and serum ferritin was found, possibly because serum ferritin, which does not cross the blood-brain barrier, is not a good marker of brain siderosis [106].

\section{Pancreas}

Iron overload leads to impairment of the endocrine and exocrine functions of the pancreas [107, 108]. Pancreatic siderosis has been evaluated in thalassaemia patients using SIR techniques and relaxometry techniques [61, 84, 109]. Midiri et al. [109], using fat as the reference tissue, found a significant negative correlation of SIR with serum ferritin and trypsin. Papakonstantinou et al. [61], using muscle as the reference tissue, found no relationship of the pancreatic SIR with the hepatic SIR or serum ferritin. The findings of Argyropoulou et al. [84], using T2 relaxometry techniques, were similar. In addition, relaxometry techniques demon- 
strated that the $\mathrm{T} 2$ relaxation time of the pancreas of patients with thalassaemia is significantly lower in children than in adults [84]. Fatty degeneration of the pancreas in adult patients may explain these differences (Fig. 5).

\section{Adrenals}

Abnormalities in adrenal function have been reported in patients with thalassaemia [103]. There is only one study evaluating adrenals for iron overload with MRI (Fig. 5), which showed a significant correlation between adrenal and liver siderosis [87].

Spleen, lymph nodes and bone marrow

In spite of the fact that the spleen, lymph nodes and bone marrow, which all contain reticuloendothelial cells, are among the first organs to be affected by iron overload [1, 13], there have been very few studies evaluating their iron overload in thalassaemia by MRI, and these used mainly SIR techniques [59, 60, 62, 84]. SIR of the spleen shows a significant correlation with serum ferritin but not with SIR of the liver (Fig. 5) [62]. The absence of correlation between liver and spleen siderosis could be explained by differences in iron kinetics, by differences in the cluster size of iron proteins, by haemochromatosis gene mutations in $\beta$-thalassaemia major carriers, and by the presence of extramedullary haemopoietic tissue in the spleen [78, 110, 111]. Intraabdominal lymph nodes in $\beta$-thalassaemia have been related to chronic hepatitis $\mathrm{C}$ [60]. Lymph node siderosis correlates with liver, but not with spleen siderosis [60].

In the few studies that have been reported, SIR and relaxometry methods have shown discordant results for MR parameters of bone marrow siderosis and serum ferritin [59, 84]. Normal bone marrow signal associated with liver siderosis has been reported in a few patients with thalassaemia and this may be due to differences in genotype or differences in chelation therapy regimens [59].

\section{Conclusion}

It is evident that MR relaxometry has the potential to become the method of choice for non-invasive, safe and accurate assessment of iron load. Further theoretical research, along with studies monitoring wider age groups of patients are needed before a generally accepted protocol can be established. Until then, extreme caution is needed in its clinical application and interpretation. Experimental artefacts, non-optimized protocols, poor data analysis and unawareness of the inherent limitations of current methodologies in assessing a heavy body iron burden can result in misleading diagnosis and inappropriate management of thalassaemia patients with iron overload. Future experimental developments in relaxometry based on a better theoretical understanding of the contribution of the iron-containing proteins to the MR signal are expected to further strengthen its clinical role in the monitoring of patients with $\beta$-thalassaemia major.

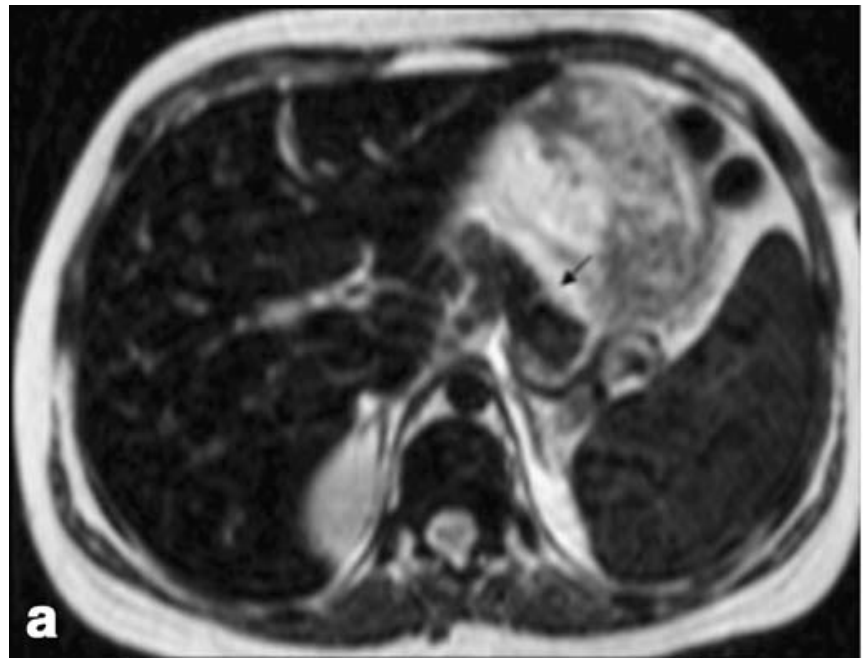

Fig. 5 Siderosis of the spleen, pancreas and adrenals. a A 14-year-old male with $\beta$-thalassaemia major. Axial scan, fourth echo of a multiecho sequence (TR/TE 2,000/20, 40, 60, 80, 100, 120, 140, $160 \mathrm{~ms}$ ) shows low-signal intensity of the liver, pancreas (arrow) and spleen suggesting increased iron deposition. b A 27-year-old-male

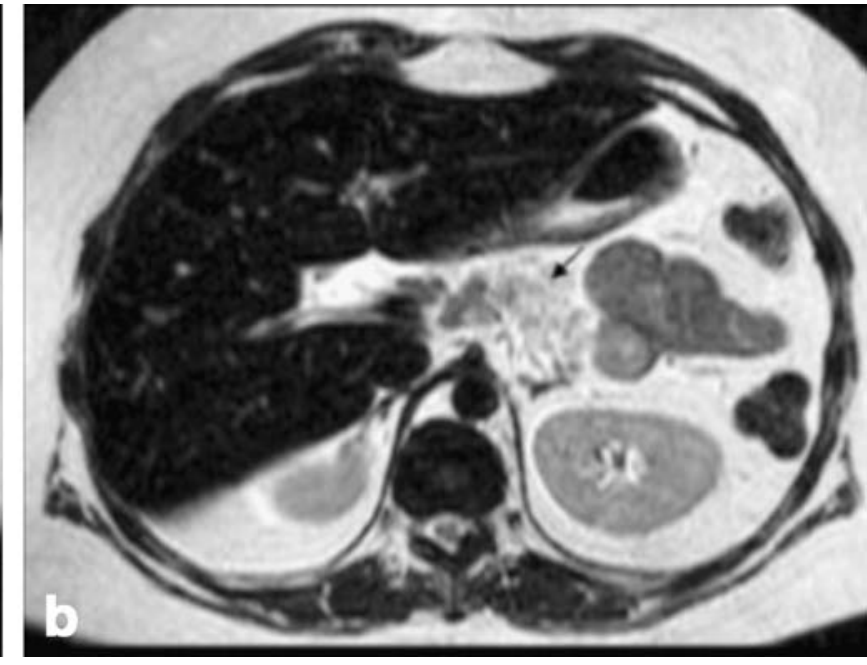

with $\beta$-thalassaemia major and diabetes. Axial T2-W (TR/TE 1,800/ $80 \mathrm{~ms}$ ) scan shows low-signal intensity of the liver and adrenals suggesting siderosis. High signal intensity of the pancreas (arrow) suggests tissue damage and fatty degeneration 


\section{References}

1. Rund D, Rachmilewitz E (2005) Beta-thalassemia. N Engl J Med 353:1135-1146

2. Forget BG (1993) The pathophysiology and molecular genetics of beta thalassemia. Mt Sinai J Med 60:95-103

3. Pootrakul P, Sirankapracha P, Hemsorach S et al (2000) A correlation of erythrokinetics, ineffective erythropoiesis, and erythroid precursor apoptosis in Thai patients with thalassemia. Blood 96:2606-2612

4. Hentze MW, Muckenthaler MU, Andrews NC (2004) Balancing acts: molecular control of mammalian iron metabolism. Cell 117:285-297

5. Gossuin Y, Muller RN, Gillis P (2004) Relaxation induced by ferritin: a better understanding for an improved MRI iron quantification. NMR Biomed 17:427-432

6. Brittenham GM (2005) Iron balance in the red blood cell donor. Dev Biol (Basel) 120:77-82

7. Porter JB, Davis BA (2002) Monitoring chelation therapy to achieve optimal outcome in the treatment of thalassaemia. Best Pract Res Clin Haematol 15:329-368

8. Cunningham MJ, Nathan DG (2005) New developments in iron chelators. Curr Opin Hematol 12:129-134

9. Hershko C, Link G, Konijn AM et al (2005) Objectives and mechanism of iron chelation therapy. Ann N Y Acad Sci 1054: 124-135

10. Chaston TB, Richardson DR (2003) Iron chelators for the treatment of iron overload disease: relationship between structure, redox activity, and toxicity. Am J Hematol 73:200-210

11. Torti FM, Torti SV (2002) Regulation of ferritin genes and protein. Blood 99:3505-3516

12. Rothman RJ, Serroni A, Farber JL (1992) Cellular pool of transient ferric iron, chelatable by deferoxamine and distinct from ferritin, that is involved in oxidative cell injury. Mol Pharmacol 42:703-710

13. Gabutti V, Piga A, Sacchetti L et al (1989) Quality of life and life expectancy in thalassemic patients with complications. Prog Clin Biol Res 309:35-41

14. Saito K, Nishisato T, Grasso JA et al (1986) Interaction of transferrin with iron-loaded rat peritoneal macrophages. $\mathrm{Br} \mathrm{J}$ Haematol 62:275-286

15. Porter JB, Rafique R, Srichairatanakool S et al (2005) Recent insights into interactions of deferoxamine with cellular and plasma iron pools: Implications for clinical use. Ann N Y Acad Sci 1054:155-168

16. Shalev O, Repka T, Goldfarb A et al (1995) Deferiprone (L1) chelates pathologic iron deposits from membranes of intact thalassemic and sickle red blood cells both in vitro and in vivo. Blood 86:2008-2013

17. Mazza P, Giua R, De Marco S et al (1995) Iron overload in thalassemia: comparative analysis of magnetic resonance imaging, serum ferritin and iron content of the liver. Haematologica 80:398-404

18. Papakonstantinou OG, Maris TG, Kostaridou V et al (1995) Assessment of liver iron overload by T2-quantitative magnetic resonance imaging: correlation of T2-QMRI measurements with serum ferritin concentration and histologic grading of siderosis. Magn Reson Imaging 13:967-977

19. Gandon Y, Olivie D, Guyader D et al (2004) Non-invasive assessment of hepatic iron stores by MRI. Lancet 363:357-362

20. Locasciulli A, Monguzzi W, Tornotti G et al (1993) Hepatitis C virus infection and liver disease in children with thalassemia. Bone Marrow Transplant 12 [Suppl 1]:18-20

21. Tong MJ, el-Farra NS, Reikes AR et al (1995) Clinical outcomes after transfusion-associated hepatitis C. N Engl J Med 332:1463-1466
22. Pennell DJ (2005) T2* magnetic resonance and myocardial iron in thalassemia. Ann N Y Acad Sci 1054:373-378

23. Kaltwasser JP, Gottschalk R, Schalk KP et al (1990) Noninvasive quantitation of liver iron-overload by magnetic resonance imaging. Br J Haematol 74:360-363

24. Chezmar JL, Nelson RC, Malko JA et al (1990) Hepatic iron overload: diagnosis and quantification by noninvasive imaging. Gastrointest Radiol 15:27-31

25. Bonkovsky HL, Slaker DP, Bills EB et al (1990) Usefulness and limitations of laboratory and hepatic imaging studies in ironstorage disease. Gastroenterology 99:1079-1091

26. Guyader D, Gandon Y, Robert JY et al (1992) Magnetic resonance imaging and assessment of liver iron content in genetic hemochromatosis. J Hepatol 15:304-308

27. Ernst O, Sergent G, Bonvarlet P et al (1997) Hepatic iron overload: diagnosis and quantification with MR imaging. AJR 168:1205-1208

28. Christoforidis A, Haritandi A, Tsitouridis I et al (2006) Correlative study of iron accumulation in liver, myocardium, and pituitary assessed with MRI in young thalassemic patients. $\mathrm{J}$ Pediatr Hematol Oncol 28:311-315

29. Kim MJ, Mitchell DG, Ito K et al (2002) Hepatic iron deposition on magnetic resonance imaging: correlation with inflammatory activity. J Comput Assist Tomogr 26:988-993

30. Gandon Y, Guyader D, Heautot JF et al (1994) Hemochromatosis: diagnosis and quantification of liver iron with gradientecho MR imaging. Radiology 193:533-538

31. Rocchi E, Cassanelli M, Borghi A et al (1993) Magnetic resonance imaging and different levels of iron overload in chronic liver disease. Hepatology 17:997-1002

32. Gomori JM, Horev G, Tamary H et al (1991) Hepatic iron overload: quantitative MR imaging. Radiology 179:367-369

33. Bartzokis G, Aravagiri M, Oldendorf WH et al (1993) Field dependent transverse relaxation rate increase may be a specific measure of tissue iron stores. Magn Reson Med 29:459-464

34. Gelman N, Gorell JM, Barker PB et al (1999) MR imaging of human brain at $3.0 \mathrm{~T}$ : preliminary report on transverse relaxation rates and relation to estimated iron content. Radiology 210:759-767

35. Ghugre NR, Enriquez CM, Coates TD et al (2006) Improved R2* measurements in myocardial iron overload. J Magn Reson Imaging 23:9-16

36. Mavrogeni SI, Markussis V, Kaklamanis L et al (2005) A comparison of magnetic resonance imaging and cardiac biopsy in the evaluation of heart iron overload in patients with betathalassemia major. Eur J Haematol 75:241-247

37. Aessopos A, Giakoumis A, Fragodimitri C et al (2007) Correlation of echocardiography parameters with cardiac magnetic resonance imaging in transfusion-dependent thalassaemia major. Eur J Haematol 78:58-65

38. Pennell D (2006) MRI and iron-overload cardiomyopathy in thalassaemia. Circulation 113:f43-44

39. Alexopoulou E, Stripeli F, Baras P et al (2006) R2 relaxometry with MRI for the quantification of tissue iron overload in betathalassemic patients. J Magn Reson Imaging 23:163-170

40. Voskaridou E, Douskou M, Terpos E et al (2004) Magnetic resonance imaging in the evaluation of iron overload in patients with beta thalassaemia and sickle cell disease. Br J Haematol 126:736-742

41. Carneiro AA, Fernandes JP, de Araujo DB et al (2005) Liver iron concentration evaluated by two magnetic methods: magnetic resonance imaging and magnetic susceptometry. Magn Reson Med 54:122-128

42. Pepe A, Positano V, Santarelli MF et al (2006) Multislice multiecho $\mathrm{T}^{*} *$ cardiovascular magnetic resonance for detection of the heterogeneous distribution of myocardial iron overload. J Magn Reson Imaging 23:662-668 
43. Argyropoulou MI, Metafratzi Z, Kiortsis DN et al (2000) T2 relaxation rate as an index of pituitary iron overload in patients with beta-thalassemia major. AJR 175:1567-1569

44. St Pierre TG, Clark PR, Chua-anusorn W et al (2005) Noninvasive measurement and imaging of liver iron concentrations using proton magnetic resonance. Blood 105:855-861

45. Brittenham GM, Badman DG (2003) Noninvasive measurement of iron: report of an NIDDK workshop. Blood 101:15-19

46. Wang ZJ, Haselgrove JC, Martin MB et al (2002) Evaluation of iron overload by single voxel MRS measurement of liver T2. J Magn Reson Imaging 15:395-400

47. Vymazal J, Urgosik D, Bulte JW (2000) Differentiation between hemosiderin- and ferritin-bound brain iron using nuclear magnetic resonance and magnetic resonance imaging. Cell Mol Biol (Noisy-le-grand) 46:835-842

48. Halle B (1999) Water in biological systems: the NMR picture. In: Bellisent-Funel M-C (ed) Hydration processes in biology. IOS Press, Amsterdam, pp 221-232

49. Tian G, Shen J, Su S et al (1997) Evaluation of hydroxyethyl-starchferrioxamine as an intravascular MR contrast agent for assessment of myocardial perfusion. Acta Radiol Suppl 412:85-90

50. Bloembergen N (1957) Proton relaxation time in paramagnetic solutions. J Chem Phys 25:572-573

51. Gueron M (1975) Nuclear relaxation in macromolecules by paramagnetic ions: a novel mechanism. J Magn Reson 19:58-66

52. Gillis P, Roch A, Brooks RA (1999) Corrected equations for susceptibility-induced T2-shortening. J Magn Reson 137:402-407

53. Gillis P, Koenig SH (1987) Transverse relaxation of solvent protons induced by magnetized spheres: application to ferritin, erythrocytes, and magnetite. Magn Reson Med 5:323-345

54. Gillis P, Moiny F, Brooks RA (2002) On T(2)-shortening by strongly magnetized spheres: a partial refocusing model. Magn Reson Med 47:257-263

55. Gossuin Y, Roch A, Muller RN et al (2002) Anomalous nuclear magnetic relaxation of aqueous solutions of ferritin: an unprecedented first-order mechanism. Magn Reson Med 48:959-964

56. Denisov V, Venu K, Peters J et al (1997) Orientational disorder and entropy of water in protein cavities. J Phys Chem B 101:9380-9389

57. Gossuin Y, Roch A, Lo Bue F et al (2001) Nuclear magnetic relaxation dispersion of ferritin and ferritin-like magnetic particle solutions: a pH-effect study. Magn Reson Med 46:476-481

58. Argyropoulou MI, Kiortsis DN, Efremidis SC (2003) MRI of the liver and the pituitary gland in patients with beta-thalassemia major: does hepatic siderosis predict pituitary iron deposition? Eur Radiol 13:12-16

59. Drakonaki EE, Maris TG, Papadakis A et al (2007) Bone marrow changes in beta-thalassemia major: quantitative MR imaging findings and correlation with iron stores. Eur Radiol 17:2079-2087

60. Papakonstantinou O, Maris TG, Kostaridou S et al (2005) Abdominal lymphadenopathy in beta-thalassemia: MRI features and correlation with liver iron overload and posttransfusion chronic hepatitis C. AJR 185:219-224

61. Papakonstantinou O, Ladis V, Kostaridou S et al (2006) The pancreas in beta-thalassemia major: MR imaging features and correlation with iron stores and glucose disturbances. Eur Radiol $17: 1535-1543$

62. Papakonstantinou O, Drakonaki EE, Maris T et al (2006) MR imaging of spleen in beta-thalassemia major. Abdom Imaging. DOI 10.1007/s00261-006-9138-4

63. St Pierre TG, Clark PR, Chua-Anusorn W (2005) Measurement and mapping of liver iron concentrations using magnetic resonance imaging. Ann N Y Acad Sci 1054:379-385

64. Fenzi A, Bortolazzi M, Marzola P (2003) Comparison between signal-to-noise ratio, liver-to-muscle ratio, and 1/T2 for the noninvasive assessment of liver iron content by MRI. J Magn Reson Imaging 17:589-592

65. Carr HY, Purcell EM (1954) Effects of diffusion on free precession in nuclear magnetic resonance experiments. Phys Rev 94:630-638

66. Meiboom S, Gill D (1958) Modified spin-echo method for measuring nuclear relaxation times. Rev Sci Instrum 29:688-691

67. In den Kleef JJ, Cuppen JJ (1987) RLSQ: T1, T2, and rho calculations, combining ratios and least squares. Magn Reson Med 5:513-524

68. Anderson LJ, Holden S, Davis B et al (2001) Cardiovascular T2star $(\mathrm{T} 2 *)$ magnetic resonance for the early diagnosis of myocardial iron overload. Eur Heart J 22:2171-2179

69. Wood JC, Tyszka JM, Carson S et al (2004) Myocardial iron loading in transfusion-dependent thalassemia and sickle cell disease. Blood 103:1934-1936

70. Jensen PD, Jensen FT, Christensen T et al (2003) Evaluation of myocardial iron by magnetic resonance imaging during iron chelation therapy with deferrioxamine: indication of close relation between myocardial iron content and chelatable iron pool. Blood 101:4632-4639

71. Westwood MA, Anderson LJ, Firmin DN et al (2003) Interscanner reproducibility of cardiovascular magnetic resonance T2* measurements of tissue iron in thalassemia. J Magn Reson Imaging 18:616-620

72. Gorell JM, Ordidge RJ, Brown GG et al (1995) Increased ironrelated MRI contrast in the substantia nigra in Parkinson's disease. Neurology 45:1138-1143

73. Ordidge RJ, Gorell JM, Deniau JC et al (1994) Assessment of relative brain iron concentrations using $\mathrm{T} 2$-weighted and $\mathrm{T} 2$ * $_{\text {- }}$ weighted MRI at 3 Tesla. Magn Reson Med 32:335-341

74. Wacker CM, Bock M, Hartlep AW et al (1999) Changes in myocardial oxygenation and perfusion under pharmacological stress with dipyridamole: assessment using $T^{*} 2$ and $\mathrm{T} 1$ measurements. Magn Reson Med 41:686-695

75. St Pierre TG (2003) Deferiprone versus desferrioxamine in thalassaemia, and $\mathrm{T} 2 *$ validation and utility. Lancet $361: 182$; author reply $183-184$

76. Bauer WR, Nadler W, Bock M et al (1999) Theory of the bold effect in the capillary region: an analytical approach for the determination of T2 in the capillary network of myocardium. Magn Reson Med 41:51-62

77. Papakonstantinou O, Kostaridou S, Maris T et al (1999) Quantification of liver iron overload by $\mathrm{T} 2$ quantitative magnetic resonance imaging in thalassemia: impact of chronic hepatitis $\mathrm{C}$ on measurements. J Pediatr Hematol Oncol 21:142-148

78. St Pierre TG, Tran KC, Webb J et al (1991) Organ-specific crystalline structures of ferritin cores in beta-thalassemia/hemoglobin E. Biol Met 4:162-165

79. Ladis V, Chouliaras G, Berdousi H et al (2005) Longitudinal study of survival and causes of death in patients with thalassemia major in Greece. Ann N Y Acad Sci 1054:445-450

80. Kattamis A, Kassou C, Berdousi H et al (2003) Combined therapy with desferrioxamine and deferiprone in thalassemic patients: effect on urinary iron excretion. Haematologica 88:1423-1425

81. Christoforidis A, Haritandi A, Perifanis V et al (2007) MRI for the determination of pituitary iron overload in children and young adults with beta-thalassaemia major. Eur J Radiol 62:138-142

82. Christoforidis A, Haritandi A, Tsatra I et al (2007) Four-year evaluation of myocardial and liver iron assessed prospectively with serial MRI scans in young patients with beta-thalassaemia major: comparison between different chelation regimens. Eur J Haematol 78:52-57

83. Sparacia G, Iaia A, Banco A et al (2000) Transfusional hemochromatosis: quantitative relation of MR imaging pituitary signal intensity reduction to hypogonadotropic hypogonadism. Radiology 215:818-823 
84. Argyropoulou MI, Kiortsis DN, Astrakas L et al (2007) Liver, bone marrow, pancreas and pituitary gland iron overload in young and adult thalassemic patients: a T2 relaxometry study. Eur Radiol. DOI 10.1007/s00330-007-0683-1

85. Wood JC, Enriquez C, Ghugre N et al (2005) MRI R2 and R2* mapping accurately estimates hepatic iron concentration in transfusion-dependent thalassemia and sickle cell disease patients. Blood 106:1460-1465

86. Aldouri MA, Wonke B, Hoffbrand AV et al (1987) Iron state and hepatic disease in patients with thalassaemia major, treated with long term subcutaneous desferrioxamine. J Clin Pathol 40:1353-1359

87. Drakonaki E, Papakonstantinou O, Maris T et al (2005) Adrenal glands in beta-thalassemia major: magnetic resonance (MR) imaging features and correlation with iron stores. Eur Radiol 15:2462-2468

88. Kushner JP, Porter JP, Olivieri NF (2001) Secondary iron overload. Hematology Am Soc Hematol Educ Program 47-61

89. Gatter KC, Brown G, Trowbridge IS et al (1983) Transferrin receptors in human tissues: their distribution and possible clinical relevance. J Clin Pathol 36:539-545

90. Atkin SL, Burnett HE, Green VL et al (1996) Expression of the transferrin receptor in human anterior pituitary adenomas is confined to gonadotrophinomas. Clin Endocrinol (Oxf) 44:467-471

91. Thorstensen K, Trinder D, Zak O et al (1995) Uptake of iron from N-terminal half-transferrin by isolated rat hepatocytes. Evidence of transferrin-receptor-independent iron uptake. Eur J Biochem 232:129-133

92. Barry M, Flynn DM, Letsky EA et al (1974) Long-term chelation therapy in thalassaemia major: effect on liver iron concentration, liver histology, and clinical progress. Br Med J 2:16-20

93. Cohen A (1987) Management of iron overload in the pediatric patient. Hematol Oncol Clin North Am 1:521-544

94. Zurlo MG, De Stefano P, Borgna-Pignatti C et al (1989) Survival and causes of death in thalassaemia major. Lancet 2:27-30

95. Borgna-Pignatti C, Rugolotto S, Nobili B et al (1997) A trial of high-dose dexamethasone therapy for chronic idiopathic thrombocytopenic purpura in childhood. J Pediatr 130:13-16

96. Mavrogeni SI, Gotsis ED, Markussis V et al (1998) T2 relaxation time study of iron overload in b-thalassemia. Magma 6:7-12

97. Jensen PD, Jensen FT, Christensen $T$ et al (2001) Indirect evidence for the potential ability of magnetic resonance imaging to evaluate the myocardial iron content in patients with transfusional iron overload. Magma 12:153-166

98. He T, Gatehouse PD, Anderson LJ et al (2006) Development of a novel optimized breathhold technique for myocardial T2 measurement in thalassemia. J Magn Reson Imaging 24:580-585
99. Economou-Petersen E, Aessopos A, Kladi A et al (1998) Apolipoprotein E epsilon4 allele as a genetic risk factor for left ventricular failure in homozygous beta-thalassemia. Blood 92:3455-3459

100. Ferrara M, Matarese SM, Francese M et al (2001) Role of apolipoprotein E (APOE) polymorphism on left cardiac failure in homozygous beta thalassaemic patients. Br J Haematol 114:959960

101. Olson LJ, Edwards WD, McCall JT et al (1987) Cardiac iron deposition in idiopathic hemochromatosis: histologic and analytic assessment of 14 hearts from autopsy. J Am Coll Cardiol 10:1239-1243

102. Bergeron C, Kovacs K (1978) Pituitary siderosis. A histologic, immunocytologic, and ultrastructural study. Am J Pathol 93:295-309

103. Oerter KE, Kamp GA, Munson PJ et al (1993) Multiple hormone deficiencies in children with hemochromatosis. J Clin Endocrinol Metab 76:357-361

104. Argyropoulou MI, Kiortsis DN, Metafratzi Z et al (2001) Pituitary gland height evaluated by MR in patients with betathalassemia major: a marker of pituitary gland function. Neuroradiology 43:1056-1058

105. Sparacia G, Midiri M, D'Angelo P et al (1999) Magnetic resonance imaging of the pituitary gland in patients with secondary hypogonadism due to transfusional hemochromatosis. Magma 8:87-90

106. Metafratzi Z, Argyropoulou MI, Kiortsis DN et al (2001) T(2) relaxation rate of basal ganglia and cortex in patients with betathalassaemia major. Br J Radiol 74:407-410

107. Kattamis C, Ladis V, Tsoussis D et al (2004) Evolution of glucose intolerance and diabetes in transfused patients with thalassemia. Pediatr Endocrinol Rev 2 [Suppl 2]:267-271

108. Gullo L, Corcioni E, Brancati C et al (1993) Morphologic and functional evaluation of the exocrine pancreas in beta-thalassemia major. Pancreas 8:176-180

109. Midiri M, Lo Casto A, Sparacia G et al (1999) MR imaging of pancreatic changes in patients with transfusion-dependent betathalassemia major. AJR 173:187-192

110. Martins R, Picanco I, Fonseca A et al (2004) The role of HFE mutations on iron metabolism in beta-thalassemia carriers. $\mathrm{J}$ Hum Genet 49:651-655

111. Katz DS (1999) Splenic extramedullary hematopoiesis: large focal lesion in a patient with thalassemia. AJR 173:1715-1716

112. Argyropoulou M, Perignon F, Brunelle F et al (1991) Height of normal pituitary gland as a function of age evaluated by magnetic resonance imaging in children. Pediatr Radiol 21:247-249 